\title{
Hierarchical Control Strategies for Energy Management of Connected Hybrid Electric Vehicles in Urban Roads
}

\author{
Baisravan HomChaudhuri, ${ }^{* 1}$ Runing $\operatorname{Lin}^{2}$ and Pierluigi Pisu ${ }^{3}$ *
}

\begin{abstract}
This paper presents a fuel efficient control strategy for a group of connected hybrid electric vehicles (HEVs) in urban road conditions. A hierarchical control architecture is proposed in this paper for every HEV, where the higher level and the lower level controller share information with each other and solve two different problems that aim at improving its fuel efficiency. The higher level controller of each $\mathrm{HEV}$ is considered to utilize traffic light information, through vehicle to infrastructure (V2I) communication, and state information of the vehicles in its near neighborhood, via vehicle to vehicle $(\mathrm{V} 2 \mathrm{~V})$ communication. Apart from that, the higher level controller of each HEV uses the recuperation information from the lower level controller and provides it the optimal velocity profile by solving its problem in a model predictive control framework. Each lower level controller uses adaptive equivalent consumption minimization strategy (ECMS) for following their velocity profiles, obtained from the higher level controller, in a fuel efficient manner. In this paper, the vehicles are modeled in Autonomie software and the simulation results are provided in the paper that shows the effectiveness of the proposed control architecture.
\end{abstract}

\footnotetext{
${ }^{* 1}$ Baisravan HomChaudhuri is a Postdoctoral Fellow of Automotive Engineering, Clemson University, Greenville, SC 29607, USA baisravan.hc@gmail.com

$\dagger^{2}$ Runing Lin is a senior engineer at China North Vehicle Research Institute, China linruning2003@tom. com

$¥ 3$ Pierluigi Pisu is with the Department of Automotive Engineering, Clemson University, Greenville, SC 29607, USA pisup@clemson.edu

1

(C) 2015. This manuscript version is made available under the Elsevier user license http://www.elsevier.com/open-access/userlicense/1.0/
} 


\section{List of Figures}

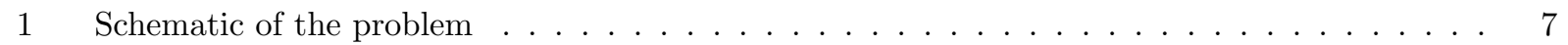

2 Battery Equivalent Circuit Model . . . . . . . . . . . . . . . . . . . . . . 9

3 Schematic of the Hierarchical Control Architecture . . . . . . . . . . . . . . . . . . 9

4 Schematic of Velocity Range and Target Velocity Calculation _ . . . . . . . . . . . . . . 11

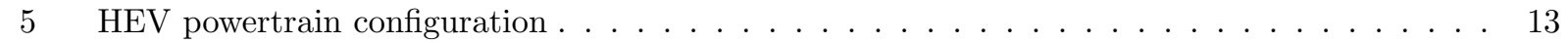

6 Comparison of Vehicle trajectories for 10 mins $\ldots \ldots \ldots \ldots \ldots \ldots \ldots$

7 Comparison of Vehicle trajectories for 5 mins $\ldots \ldots \ldots \ldots \ldots$

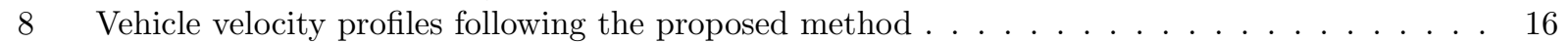

9 Vehicle velocity profiles following the baseline method $(\mathrm{CCC}) \ldots \ldots \ldots \ldots$

10 Vehicle trajectories in the heterogeneous scenario with vehicle index 5,7 and 9 are regular

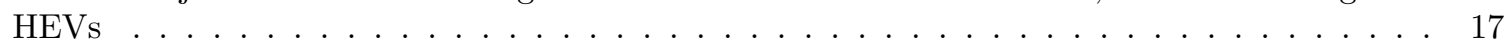

11 Lower Level Control: Vehicle $1 \ldots \ldots \ldots \ldots$

12 Lower Level Control: Vehicle $2 \ldots \ldots \ldots \ldots$

13 Lower Level Control: Vehicle $3 \ldots \ldots$. . . . . . . . . . . . . . . . . . . 19

14 Lower Level Control: Vehicle $4 \ldots \ldots \ldots$

15 Lower Level Control: Vehicle $5 \ldots \ldots \ldots$

16 Lower Level Control: Vehicle $6 \ldots \ldots \ldots$

17 Lower Level Control: Vehicle $7 \ldots \ldots \ldots \ldots$

18 Lower Level Control: Vehicle $8 \ldots \ldots \ldots \ldots \ldots \ldots$

19 Lower Level Control: Vehicle $9 \ldots \ldots \ldots \ldots$

20 Lower Level Control: Vehicle $10 \ldots \ldots \ldots \ldots$. . . . . . . . . . . . . . . 21 


\section{List of Tables}

1 Components and Parameters of the Vehicle . . . . . . . . . . . . . . . . . . 14

2 Comparison between the Baseline Method and the Proposed Method . . . . . . . . . . . . 15

3 Heterogeneous Scenario with Both Regular and Connected Vehicles . . . . . . . . . . . . . 18 
Keywords - Connected Vehicles, Model Predictive Control, Adaptive Equivalent Consumption Minimization Strategy, Intelligent Transportation System, Hybrid Electric Vehicles 


\section{INTRODUCTION}

Connected vehicle technology proposed by the Intelligent Transportation Systems Joint Program Office (ITSJPO) of the United States Department of Transportation addresses some of the issues associated with the current transportation system. In the connected vehicle system, vehicles are capable of communicating with other vehicles (vehicle to vehicle communication (V2V)) and transportation infrastructure (vehicle to infrastructure communication (V2I)) via wireless communications such as dedicated short range communications (DSRC). In the current transportation system, the major areas of concern are mobility, environmental impacts and safety. The primary aim of the envisioned connected vehicle technology/systems is to improve safety of the transportation system since it has been reported that V2V and V2I communication addresses almost $79 \%$ of all vehicle crashes (Najm et al. (2010)). According to the ITS-JPO website, traffic congestion costs 87.2 billion on the U.S. economy, with 4.2 billion hours and 2.8 billion gallons of fuel spent sitting in traffic. Moreover, deterioration of mobility affects the environment since it has been seen that vehicles that are stationary, idling, and traveling in a stop-and-go pattern due to congestion emits more greenhouse gases than those traveling in free-flow conditions.

Hybrid electric vehicles (HEVs) have attracted a lot of attention in the last decade because of their fuel efficiency and emission. HEVs improve fuel efficiency by using power from both the energy storage systems (batteries) and the engine, and by using regenerative braking. At any time, the power split between the engine and the battery is required to be optimized to improve fuel efficiency while satisfying the driver power request. The control strategies for HEVs available in the literature can be categorized as dynamic programming based approach (Lin et al. (2001), Brahma et al. (2000)), artificial intelligence based approach (Salman et al. (2000)) and methods based on electric power to equivalent fuel conversion (Paganelli et al. (2001, 2000), Onori et al. (2010)). It has been pointed out in Brahma et al. (2000) that knowledge of future driving profile further improves vehicle fuel efficiency. Some of the literature considering future driving behavior and road conditions for HEVs as well as for plug-in electric vehicles are available in Vogel et al. (2012), Zhang \& Vahidi (2011), Zhang et al. (2010, 2009), Gong et al. (2008), Sun et al. (2015). Authors in Zhang \& Vahidi (2011), Zhang et al. (2010, 2009) have developed energy management strategies for both HEVs and plug-in electric vehicles that uses real-time road grade and trip information.

Future driving behavior prediction using historical data is available in Vogel et al. (2012), Gong et al. (2008), Sun et al. (2015), where a reinforcement learning based driver model is developed in Vogel et al. (2012) to predict future driving behavior while data driven trip information is developed in Gong et al. (2008) and Sun et al. (2015) that predicts future vehicle velocities for plug-in electric and plug-in hybrid electric vehicles respectively. The future velocities in Gong et al. (2008) and Sun et al. (2015) are predicted using average road velocity data and the power split is performed using dynamic programming and model predictive control strategy respectively. The predicted velocities in Gong et al. (2008) and Sun et al. (2015) are approximate average vehicle velocities that are computed at a macro level of the transportation system and do not consider micro level details such position of preceding vehicles that influences vehicle velocities to a great extent (collision avoidance). Most of these research only focus on controlling the power split between the engine and the battery and do not consider controlling the driver behavior. In this paper, we present a novel hierarchical control architecture for HEVs in urban road conditions in a connected vehicle scenario that uses both V2V and V2I communication to develop fuel efficient control strategies by controlling both the vehicle driving behavior as well as lower level power split.

The data rich environment developed through the process of V2V and V2I communication can be used to improve not only safety but also various other aspects of the transportation system, such as mobility and fuel economy. In recent years, a lot of research in automotive engineering and intelligent transportation systems has focused in the development of fuel economic control strategies for conventional vehicles (Kamal et al. (2010, 2013), Chang \& Morlok (2005), Yang \& Jin (2014), Mensing et al. (2014)). The fuel efficiency of a vehicle can be improved through a number of different ways such as improvement of engine characteristics, vehicle structure against aerodynamic drag, and powertrain system. Apart from these factors, fuel efficiency of vehicles has also been seen to depend on the driving behavior as explained in Van Mierlo et al. (2004). The general trend in developing fuel efficient control schemes is to minimize sharp vehicle accelerations while some literatures (Kamal et al. (2010, 2013)) consider approximate fuel consumption models, as a function of vehicle velocity and acceleration, or vehicle's internal characteristics (Chang \& Morlok (2005)). Authors in Yang \& Jin (2014) provided an eco-driving approach by using velocity smoothing technique while an 
A number of literatures consider traffic lioht information for devioping fuel efficient control strates. in urban roads (Asadi \& Vahidi (2011), Mahler \& Vahidi (2014), De Nunzio et al. (2013), He et al. (2015), Mandava et al. (2009)). Authors in Mandava et al. (2009) provided an algorithm that minimizes acceleration profile and avoids stopping at red lights. A model predictive control strategy minimizing red light idling and sharp accelerations is shown in Asadi \& Vahidi (2011) while a probabilistic approach is used in Mahler \& Vahidi (2014) considering noisy traffic light conditions. A multi stage optimal control approach considering vehicle queue for multiple intersections is presented in He et al. (2015). Authors in De Nunzio et al. (2013) provided a pruning and graph discretization method for energy minimization of electric vehicles.

Most of the literature in fuel economic or energy efficient control of vehicles consider single vehicle scenario and do not consider multiple vehicles in the road where behavior of one vehicle affects the other. Although some literature considers congested traffic (Treiber et al. (2000)), they are mostly focused on safety rather than fuel economy or energy efficiency. In this paper, an effort has been made to develop fuel efficient control strategy for the individual vehicles considering congested traffic and traffic lights which are common in urban road conditions. In one of our previous research in HomChaudhuri et al. (2015), we have developed fuel economic control strategies for a group conventional vehicles in urban road conditions. In this paper, we extend our work to HEVs and develop a hierarchical control strategy that improves the performance of the group of connected HEVs in urban road conditions.

In our proposed hierarchical control architecture, the higher level controller develops the optimal velocity profile of a HEV in a model predictive control framework utilizing recuperation efficiency information from its lower level controller, and traffic light conditions (V2I information) and neighboring vehicle information (V2V communication). The optimal velocity profile (developed by the higher level controller) focuses on minimizing the average tractive energy consumption of the vehicle and the improvement of vehicle mobility by reducing red light idling. The lower level controller tracks the velocity profile obtained from the higher level controller by optimally splitting power between the vehicle engine and the battery by using an adaptive equivalent consumption minimization strategies (Sciarretta \& Guzzella (2007), Onori et al. (2010)). In this way, both the controllers work together in improving vehicle fuel efficiency. Charge sustaining parallel HEVs are considered in this paper and the software Autonomie (Michaels et al. (2010)) has been used for modeling and simulation purposes.

The paper contributions can be highlighted as: First a novel decentralized hierarchical control architecture is presented in this paper that provides a holistic approach for improving fuel efficiency of a group of HEVs. The decentralized nature of our algorithm makes it highly scalable. Second, the paper focuses on urban road conditions and develops control strategies in presence of congestion where the behavior of one vehicle influences the other. Finally, the developed control strategies focus on reducing red light idling and vehicle safety, and thus improves system mobility which in turn reduces environmental impact of the vehicles.

The paper is organized as follows: the mathematical description of the problem is provided in the next section which is followed by the description of the approach undertaken in solving the problem. This is followed by the simulation results and conclusions.

\section{PROBLEM FORMULATION}

In this paper, we focus on a connected vehicle scenario with multiple vehicles in congested urban road conditions. In this connected vehicle framework, the information of position and velocity of a particular vehicle is considered to be available to the vehicle's near neighborhood through vehicle-to-vehicle (V2V) communication. We consider a single lane road in this paper consisting of road side units (RSUs) which possesses the information of signal phase timings of traffic lights as well as future road grade conditions. The RSUs communicate with each vehicle through the wireless DSRC network. Figure 1 shows a schematic of the mentioned scenario. In this paper, we consider a hierarchical control structure and we separate the fuel efficient control problem into two parts: (i) Higher Level Problem: to develop control strategies, traction or braking force, which minimizes vehicle average tractive energy consumption and red light idling, and (ii) Lower Level Problem: to develop control strategies, power split between the engine and the battery, that minimizes equivalent fuel consumption. 


\subsection{Higher Level Problem}

The longitudinal vehicle dynamics of any vehicle $i$ is given by Kamal et al. (2013):

$$
\begin{aligned}
\dot{x}_{i} & =f_{i}\left(x_{i}, u_{i}\right) \\
f_{i}\left(x_{i}, u_{i}\right) & =\left[\begin{array}{c}
v_{i} \\
-\frac{1}{2 M_{h}^{i}} C_{D} \rho_{a} A_{v}^{i} v_{i}^{2}-\mu g-g \theta+u_{i}
\end{array}\right]
\end{aligned}
$$

Here $x_{i}=\left[s_{i}, v_{i}\right], s_{i}$ and $v_{i}$ are the position and velocity of any vehicle $i$. At this level, the control strategy, $u_{i}$, of a vehicle $i$ at any instant is its traction or braking force per unit mass. In the above equation, $M_{h}^{i}$, $C_{D}, \rho_{a}, A_{v}^{i}, \mu$ and $\theta$ are the mass of the vehicle, drag coefficient, the air density, the frontal area of the vehicle, the rolling friction coefficient and the road gradient respectively. We consider small $\theta$ and use the approximation $\cos (\theta)=1$ and $\sin (\theta)=\theta$.

In this level of the problem, our goal is to minimize the fuel consumption per unit distance for each vehicle by controlling the vehicle traction and braking force. This is given by $\frac{\sum \dot{m}_{f u e l}^{i}(t) \Delta t}{s_{i}^{t_{f}}}$ where $\dot{m}_{f u e l}^{i}(t)$ is the rate of fuel consumption and $s_{i}^{t_{f}}$ is the total distance traveled by vehicle $i$ and $t_{f}$ is the final time. The overall problem is given by the following equation:

$$
\begin{aligned}
& \underset{u_{i}(t)}{\operatorname{argmin}} \sum_{i=1}^{n} \frac{1}{s_{i}^{t_{f}}} \sum_{t=0}^{t_{f}} \dot{m}_{\text {fuel }}^{i}(t) \Delta t \\
& \dot{m}_{\text {fuel }}^{i}(t)=\frac{1}{\eta_{e f f} H_{L H V}^{i}} P_{w}^{i}(t) \\
& P_{w}^{i}(t)= \alpha_{i} v_{i}(t)^{3}+M_{i} g v_{i}(t)(\mu+\theta) \\
&+\beta_{1}^{i}\left[v_{i}(t) M_{i} u_{i}(t)\right]+\beta_{2}^{i}\left[-\eta_{\text {rec }}(t) M_{i} u_{i} v_{i}(t)\right] \\
& \alpha_{i}= \frac{1}{2} \rho_{a} C_{d} A_{v}^{i} \\
& \beta_{1}^{i}= \begin{cases}0 & \text { if } u_{i} \leq 0 \\
1 & \text { otherwise }\end{cases} \\
& \beta_{2}^{i}= \begin{cases}0 & \text { if } u_{i}>0 \\
1 & \text { otherwise }\end{cases} \\
& v_{\text {min }} \leq v_{i}(t) \leq v_{\text {max }} \\
& u_{\text {min }}^{i} \leq u_{i}(t) \leq u_{\text {max }}^{i}
\end{aligned}
$$

In the above equation, $\dot{m}_{f u e l}$ is the rate of fuel consumption, $P_{w}^{i}$ is the power associated with the traction force, $\eta_{r e c}(t)$ is the recuperation efficiency obtained from the lower level controller and $H_{L H V}^{i}$ is the fuel lower heating value of vehicle $i$. It can be seen from Eq. (2b) that the rate of fuel consumption, following Guzzella \& Sciarretta (2007), Zhang \& Mi (2011), is considered to be a linear function of the vehicle traction power where $\eta_{\text {eff }}$ can be considered to capture efficiency of the path from the fuel tank to the vehicle powertrain. The fuel consumed while vehicle idling is neglected in this paper since we are focusing on the parallel HEVs. The constraints in Eq. (2f) and Eq. (2g) indicates the constraints on the velocity (road speed limit) and 
the acceleration. In Eq. (2f), $v_{\min }$ and $v_{\max }$ are the minimum and maximum allowable speed at a given road respectively, and $u_{\min }^{i}$ and $u_{\max }^{i}$ are the minimum and maximum possible acceleration of a vehicle $i$ respectively. It may be noted that for HEVs, the power associated with the tractive force can be supplied by both the engine and the vehicle battery and hence it can be considered that the higher level controller minimizes the upper bound of the fuel consumed per unit distance. The solution of the above problem generates the velocity profile, $v_{i}(t), \forall t$, for each vehicle $i$ which is used as an input to the lower level problem.

\subsection{Lower Level Problem}

The velocity profile obtained from solving the higher level problem can be mapped to power requirement (power demand) and in the lower level problem we focus on developing control strategies that satisfies the power demand by minimizing the equivalent fuel consumption. For a charge sustaining parallel HEV, the problem can be mathematically expressed as Kim et al. (2011), Onori et al. (2010), Serrao et al. (2009) :

$$
\begin{aligned}
\underset{u_{i}^{l}(t)}{\operatorname{argmin}} J_{i}\left(S O C_{i}(t), u_{i}^{l}(t)\right) & =\sum_{t=0}^{t_{f}} \dot{m}_{f}\left(u_{i}^{l}(t)\right) \Delta t \\
S \dot{O}_{i}(t) & =f_{\text {soc }}\left(S O C_{i}(t), u_{i}^{l}\right) \\
S O C_{i}\left(t_{f}\right) & =S O C_{i}(0) \\
S O C_{\text {min }}^{i} & \leq S O C_{i}(t) \leq S O C_{\text {max }}^{i}, \forall t \\
P_{b, \text { min }}^{i}(t) & \leq u_{i}^{l}(t) \leq P_{b, \text { max }}^{i}(t), \forall t
\end{aligned}
$$

In the above equations, $\dot{m}_{f}\left(u_{i}^{l}(t)\right)$ is the rate of fuel consumption while the control variable $u_{i}^{l}(t)$ is the power drawn from the battery of the HEV which satisfies the limits on the available battery power as shown in Eq. (3e). $P_{b, \min }^{i}(t)$ and $P_{b, \max }^{i}(t)$ are the minimum and maximum available power from the battery at any time $t$ respectively. Since we are considering charge sustaining parallel HEVs in this paper, the initial and the final state of charge (SOC) of the battery at the end of the driving cycle should be same and is shown by constraint in Eq. (3c). This means the battery power used by the HEV, resulting in reduction in battery SOC, should be compensated with charging the vehicle using engine power or regenerative braking at any time of the driving cycle. It may noted here that satisfying the constraints in Eq. (3c) in an exact manner is very hard and in many cases impossible. That is why this constraint is generally relaxed for practical solution purposes (this would be shown in the next section). Eq. (3d) shows the limits on the SOC over the total driving cycle while Eq. (3b) describes the state dynamics, $f_{s o c}\left(S O C, u_{i}^{l}\right)$, for the lower level problem where system state is the battery SOC. Modeling the battery with equivalent circuit model (Onori et al. (2010)), the state dynamics at the lower level problem is given by:

$$
\begin{aligned}
& S \dot{O} C_{i}(t)=f_{s o c}\left(S O C_{i}(t), u_{i}^{l}\right) \\
& S \dot{O} C_{i}(t)=-\frac{I}{Q_{\max }} \\
& =-\frac{1}{Q_{\max }} \frac{\left[V_{o c}\left(S O C_{i}(t)\right)+\sqrt{V_{o c}\left(S O C_{i}(t)\right)^{2}-4 P_{b a t t} R_{e q}\left(S O C_{i}(t)\right)}\right]}{2 R_{e q}}
\end{aligned}
$$

$I$ and $Q_{\max }$ in the above equations are the charging or the discharging current through the battery and the total capacity of the battery respectively. $V_{o c}\left(S O C_{i}(t)\right)$ and $R_{e q}\left(S O C_{i}(t)\right)$ are the open circuit voltage and the equivalent resistance of the battery model, shown in Fig. 2, respectively which are functions of the SOC of the battery. $P_{\text {batt }}$ is the power drawn from the battery.

\section{APPROACH}

In this section, the decentralized control strategy for each vehicle is presented. The decentralized nature of the control stems from the fact that every vehicle evaluates its own control strategy using only local information. The overall control strategy is divided into two levels, higher level and lower level controllers. The higher level controller generates the vehicle velocity profiles that minimizes average tractive energy consumption 


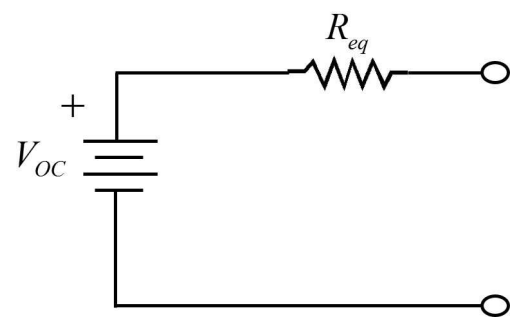

Figure 2: Battery Equivalent Circuit Model

and avoid stopping at red lights to improve system efficiency and mobility. Each lower level controller of the HEVs uses the velocity profile (from the higher level controller) as the input and generates its own fuel efficient power split control strategy. Fig. 3 shows the schematic of the hierarchical control architecture where the road side units (RSUs) share information, such as traffic light timings and road grade, to each vehicle (via V2I communication) while the vehicles also share information, such as vehicle state trajectories, with each other via V2V connectivity. It can be seen in Fig. 3 that within each vehicle, the higher level and the lower controller share information with each other to improve the performance of individual vehicles.

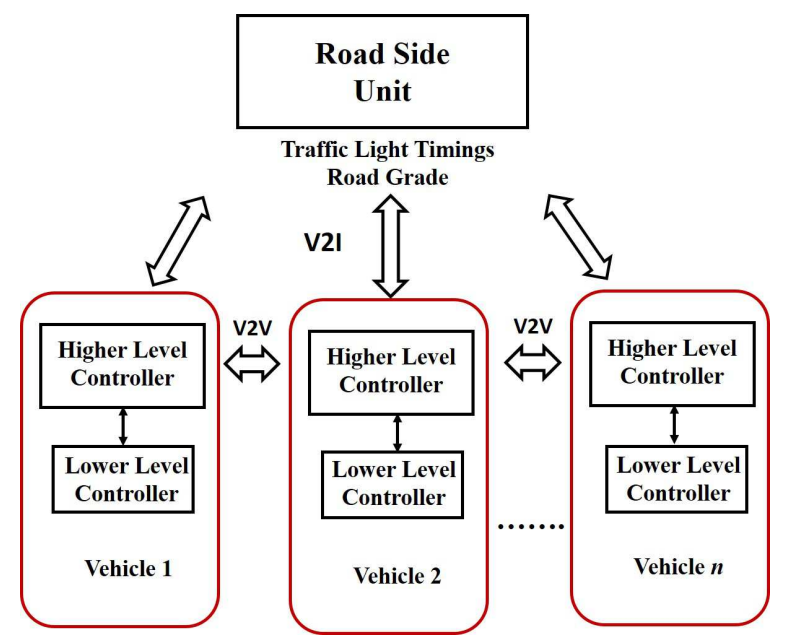

Figure 3: Schematic of the Hierarchical Control Architecture

\subsection{Higher Level Controller}

In this subsection, we describe the control strategy used to solve the higher control level problem, as described in Section 2.1, that minimizes every vehicle's average traction energy. Apart from that, since we are considering urban road conditions, the control strategy should address the issues associated with traffic light timings and road congestion. It has been seen in the literatures (Asadi \& Vahidi (2011), Mahler \& Vahidi (2014), De Nunzio et al. (2013), Mandava et al. (2009)) that avoiding stopping at red lights improve fuel efficiency and provides free flow traffic which improves system mobility and hence reduces greenhouse gas emissions. This strategy is adopted in this paper so that every vehicle would tend to avoid stopping at traffic lights. The proposed algorithm works in two phases: First a target velocity is evaluated which would enable a vehicle avoid stopping at red light, and second, a model predictive control (Grüne \& Pannek (2011)) strategy is proposed which minimizes the modeled cost function. Model predictive control is a very popular control strategy for solving constrained optimal control problems and is widely adopted in the industry (Camacho \& Alba (2013), Qin \& Badgwell (2003)). 


\subsubsection{Target Velocity}

The traffic light timings of only the approaching traffic signals are considered to be known by each vehicle and it is assumed to be noisy in nature. To avoid the noise in the traffic light timings, a green light interval $\left(t_{g}\right)$ is considered which is less than the original green light time interval $\left(t_{g}<t_{g}^{\text {original }}\right)$ so that the probability of getting a green light at the traffic signal within that period is almost 1 .

Before running the model predictive control, the target/desired velocity of a vehicle is first chosen. Rather than choosing the target or desired velocity as the maximum allowable velocity of a road as in Kamal et al. (2013), the target velocity can be chosen wisely using signal phase and timing (SPAT) information that helps the vehicle avoid stopping at red light. Thus, at time instant $k$ each vehicle is required to choose a target velocity $\left(v_{\text {target }}^{i}(k)\right)$ which is computed as Asadi \& Vahidi (2011):

$$
\begin{aligned}
& v_{\text {target }}^{i}(k)= \begin{cases}\frac{d_{i a}(k)}{K_{w} t_{c y c l e}-t_{g}-k} & \text { if light }=\text { red } \\
v_{\text {max }} & \text { if light }=\text { green and } \frac{d_{i a}(k)}{K_{w} t_{\text {cycle }}-k} \leq v_{\text {max }} \\
\frac{d_{i a}(k)}{K_{w} t_{\text {cycle }}+t_{r}-k} & \text { if light }=\text { green and Otherwise }\end{cases} \\
& \text { light }= \begin{cases}\text { red } & \text { if } 0 \leq \bmod \left(\frac{k}{t_{\text {cycle }}}\right) \leq t_{r} \\
\text { green } & \text { if } t_{r}<\bmod \left(\frac{k}{t_{\text {cycle }}}\right)<t_{\text {cycle }}\end{cases} \\
& \text { such that } v_{\min } \leq v_{\text {target }}^{i}(k) \leq v_{\max } \\
& t_{\text {cycle }}=t_{r}+t_{g} \\
& K_{w}>\frac{k}{t_{\text {cycle }}}
\end{aligned}
$$

Here $d_{i a}(k)$ is the distance between $s_{i}(k)$ (location of the $i^{t h}$ vehicle) and the traffic signal $a, t_{r}$ and $t_{g}$ are the red and green light durations respectively so that the total cycle duration is $t_{c y c l e} . K_{w}$ is an integer describing the traffic light cycle number. The function $\bmod ()$ is a modulo function which generates the residue of division $k$ by $t_{c y c l e}$. It can be seen from Eq. (5a) that when traffic light status is green, the maximum allowable speed is chosen as the target velocity unless the constraint $\frac{d_{i a}(k)}{K_{w} t_{c y c l e}-k} \leq v_{\max }$ is not satisfied. Violation of this constraint (when the signal status is green) means that the vehicle needs to break the speed limit to pass through the traffic light in the present green light window. In that case, the vehicle desires to pass through the traffic signal in the next green light window as shown in third case of Eq. (5a). If no feasible velocity is obtained in the consecutive green light windows, the vehicle has to stop at a given traffic light signal. Eq. (5e) shows the constraint on the traffic signal index number so that $K_{w}$ is increased by 1 at $k=K_{w} t_{c y c l e}$. In short, the target velocity is a feasible velocity (given by Eq. (5c)) which is chosen to make a vehicle move past a traffic signal, $a$, through a green light window.

Now, a vehicle can pass through a traffic signal through a green light window (a time range) which means there exists a range of velocities $\left[v_{l b}^{i}(k), v_{u b}^{i}(k)\right]$ which allows the vehicle avoid red light idling. Here $v_{l b}^{i}(k)$ is the lower limit while $v_{u b}^{i}(k)$ is the upper limit of the velocity range within which a vehicle $i$ can pass through a traffic light without stopping. The velocity range is chosen as:

$$
\begin{aligned}
v_{l b}^{i}(k) & = \begin{cases}\frac{d_{i a}(k)}{K_{w} t_{c y c l e}-k} & \text { if light }=\text { red } \\
\frac{d_{i a}(k)}{K_{w} t_{c y c l e}-k} & \text { if light }=\text { green and } \frac{d_{i a}(k)}{K_{w} t_{c y c l e}-k} \leq v_{\text {max }} \\
\frac{d_{i a}(k)}{\left(K_{w}+1\right) t_{c y c l e}-k} & \text { if light }=\text { green and Otherwise }\end{cases} \\
v_{u b}^{i}(k) & =v_{\text {target }}^{i}(k) \\
\text { such that } v_{\text {min }} & \leq v_{l b}^{i}(k) \leq v_{\text {target }}^{i}(k)
\end{aligned}
$$

Fig. 4 shows the schematic of velocity range and target velocity calculation where $t_{1}$ and $t_{2}$ are the time remaining for the next green signal and next red signal respectively. It can seen from Eq. (6b) that the target velocity is the upper bound of the velocity range. From this velocity range we compute the limits on the control input so that the vehicle velocity always stays within this range to avoid red light idling. 
Considering a time step of $\Delta t$, the limits of the control input can be chosen:

$$
\begin{aligned}
v_{i}(t) & \leq v_{u b}^{i}(k) \\
v_{i}(t-1)+\breve{a} \Delta t & \leq v_{u b}^{i}(k) \\
\breve{a}=u_{i}(t)+\hat{p}_{i} & \leq\left(\frac{v_{u b}^{i}(k)-v_{i}(t-1)}{\Delta t}\right) \\
\text { where } \hat{p}_{i}= & -\frac{1}{2 M_{h}} C_{D} \rho_{a} A_{v} v_{i}^{2}-\mu g-g \theta \\
\text { similarly, } u_{i}(t)+\hat{p}_{i} & \geq\left(\frac{v_{l b}^{i}(k)-v_{i}(t-1)}{\Delta t}\right)
\end{aligned}
$$

Thus from the above relations, we can evaluate the limits to control input as:

$$
\begin{aligned}
\left(\frac{v_{l b}^{i}(k)-v_{i}(t-1)}{\Delta t}\right) & -\hat{p}_{i} \leq u_{i}(t) \\
& \leq\left(\frac{v_{u b}^{i}(k)-v_{i}(t-1)}{\Delta t}\right)-\hat{p}_{i}
\end{aligned}
$$

such that $u_{\min }^{i} \leq u_{i}(t) \leq u_{\max }^{i}$

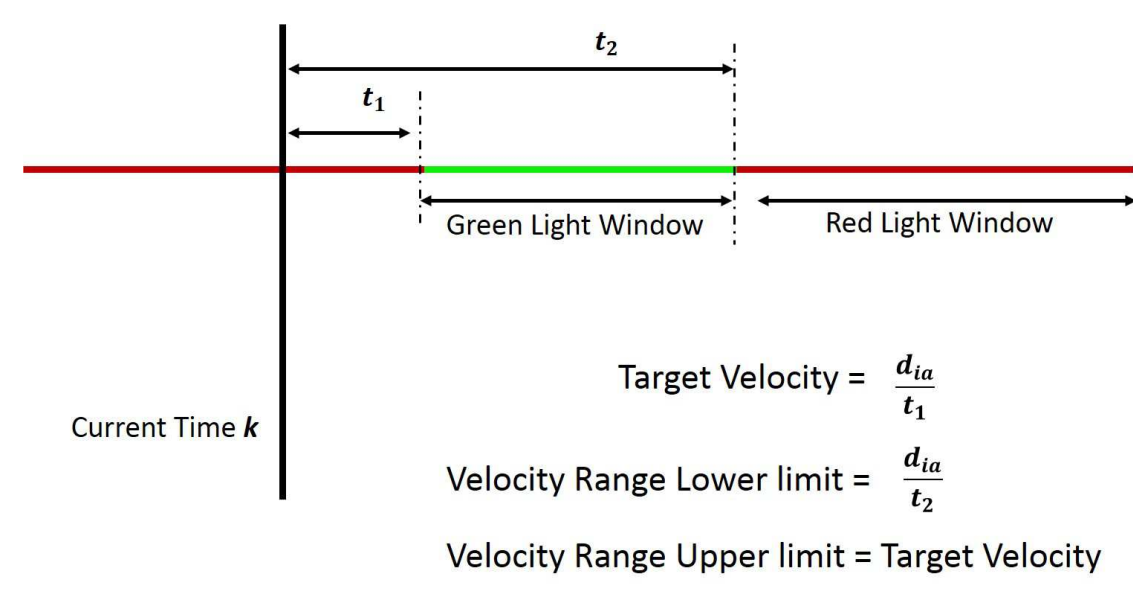

Figure 4: Schematic of Velocity Range and Target Velocity Calculation

This limit on the control input suggests that if the control is bounded by Eq. (7), the velocity of the vehicle would be bounded by $\left[v_{l b}^{i}(k), v_{u b}^{i}(k)\right]$, and thus the vehicle would be able to avoid stopping at red light.

\subsubsection{Model Predictive Control}

In this paper, we focus on developing a decentralized control strategy for individual vehicles that would result in the improvement of fuel efficiency and mobility for a group of vehicles. In the previous section, we have discussed the V2I information exchange between the traffic lights and every vehicle and the calculation of target velocity. Here we consider V2V information exchange where every vehicle, $j$, sends its state trajectory information (position information) over its future finite time horizon, $T$, to the vehicle $i$ at its immediate back. The position information over the future time horizon is obtained by solving the optimal control problem, which will be discussed later, over a finite horizon.

Once the target velocity of a vehicle has been determined and the vector of position information from its preceding vehicle is available, the problem of generation of energy efficient velocity profiles is solved in a model predictive control framework. For each vehicle $i$ and a finite time horizon $T$ the following problem, 
at time instant $k$, is being solved:

$$
\begin{aligned}
J_{i}(k) & =\underset{u_{i}(t)}{\operatorname{argmin}}\left[\sum _ { t = k } ^ { k + T - 1 } \left[w_{1} \frac{\dot{m}_{\text {fuel }}^{i}(t) \Delta t}{s_{i}(k+T-1)-s_{i}(k)}+w_{2}^{i}(t) R_{i j}(t)^{2}\right.\right. \\
& \left.+w_{3}\left(v_{i}(t)-v_{\text {target }}^{i}(k)\right)^{2}+w_{4} u_{i}(t)^{2}\right] \\
v_{l b}^{i}(k) & \leq v_{i}(t) \leq v_{u b}^{i}(k), \forall t \\
\hat{u}_{\text {min }}^{i} & \leq u_{i}(t) \leq \hat{u}_{\text {max }}^{i}, \forall t \\
R_{i j}(t) & =S_{0}+t_{h d}\left(v_{i}(t)-v_{j}(t)\right)+\left(s_{i}(t)-s_{j}(t)\right)
\end{aligned}
$$

The first term in the cost function in Eq. (8a) minimizes the fuel consumption per unit distance for the horizon $T$ where $\dot{m}_{\text {fuel }}(t)$ can be computed from Eq. (2b) and Eq. (2c), the second term minimizes the deviation from a desirable distance between vehicle $i$ and its preceding vehicle $j$, the third term tries to take the velocity of $i$ to its target velocity while the last term minimizes the control effort (throttle or braking). The new velocity (Eq (8b)) and control limits (Eq (8c)) are the evaluated from Eq. (6) and Eq. (7) respectively. The terms $S_{0}$ and $t_{h d}$ in Eq. (8d) are predefined critical distance and headway time respectively.

It may be noted that the optimum fuel efficient velocity will be very likely different from the vehicle target velocity since the latter is chosen to avoid red light idling without considering engine operation point. The solution of the problem in Eq. (8) tries to find the best velocity profile that considers both fuel efficiency and the target velocity tracking (as well as the other parts of the cost function) and the solution would depend on the weights $w_{1}$ to $w_{4}$. Following Kamal et al. (2013), $w_{2}(t)$ is chosen as a function of the relative distance, $\left(s_{j}(t)-s_{i}(t)\right)$, so that it increases as the relative distance decreases and vice versa and $w_{4}$ is a constant weight. The weights $w_{1}$ and $w_{3}$ are chosen as a function of $\left(v_{u b}^{i}(k)-v_{l b}^{i}(k)\right)$ so that when the difference is high more emphasis is given to fuel efficiency than velocity tracking ( $w_{1}$ high and $w_{3}$ low) and when the difference is low more emphasis is given to target velocity tracking ( $w_{3}$ high and $w_{1}$ low). Thus the velocity range $\left[v_{l b}^{i}(k), v_{u b}^{i}(k)\right]$ serves two purposes, (i) it helps in choosing some of the weights of the cost function, and (ii) if optimum fuel efficient velocity is far from the target velocity, it keeps the velocity deviation (from target velocity) within limits that avoid red light idling.

In this paper, we use the widely used sequential quadratic programming (Boggs \& Tolle (1995)) for solving the non-linear optimization problem described in Eq. (8). From the optimal solution of the finite horizon problem, the optimal state trajectory is also obtained and that information is shared to the immediate next vehicle. We use the 'warm start' approach for the model predictive control (Wang \& Boyd (2010)), where the optimal solution for time $k$ is used to initialize the decision variables for time instant $k+1$, to aid fast convergence of the optimization problem.

\subsection{Lower Level Control}

The lower level controller maps the velocity profile obtained from the higher level controller to power request and solves the optimal power split between the engine and the battery. Figure 5 shows the schematic of a HEV powertrain configuration. The optimal control problem in this level consists of the SOC of the battery as the system state and the battery power as the control variable as shown in Eq. (3). Solving the problem using dynamic programming is very time consuming and impractical for real-time implementations. To alleviate this problem, Energy Consumption Minimization Strategy (ECMS) (Paganelli et al. (2001, 2000)) has been widely used in the literature which, as pointed out by authors in Onori et al. (2010), Serrao et al. (2011, 2009), Kim et al. (2011), has its similarity with the Pontryagin's minimum principle (PMP) (Geering (2007)). The solution to the problem is described by the following equations where $\lambda(t)$ is the co-state of the system.

$$
\begin{aligned}
& \mathcal{H}\left(S O C_{i}, u_{i}^{l}\right)=\dot{m}_{f}\left(u_{i}^{l}(t)\right)+\lambda(t) f_{s o c}\left(S O C_{i}(t), u_{i}^{l}(t)\right) \\
& u_{i}^{l^{*}}=\underset{u_{i}^{l}}{\operatorname{argmin}} \mathcal{H}\left(S O C_{i}(t), u_{i}^{l}(t)\right) \\
& \dot{\lambda}(t)=-\frac{\delta \mathcal{H}\left(S O C_{i}(t), u_{i}^{l}\right)}{\delta S O C_{i}}=-\lambda(t) \frac{\delta S O C_{i}}{\delta S O C_{i}}
\end{aligned}
$$




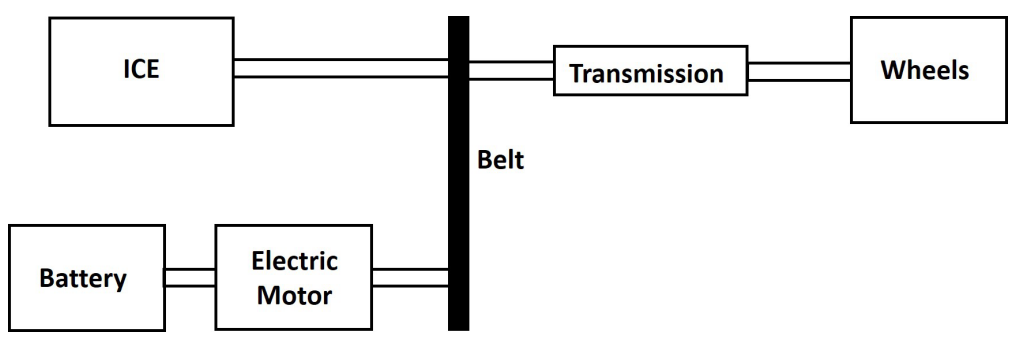

Figure 5: HEV powertrain configuration

The equivalent fuel consumption is similar to the Hamiltonian function $\mathcal{H}()$ (Onori et al. (2010)) in the equation above and thus minimization of the equivalent fuel consumption results into the minimization of the above problem. For charge sustaining HEVs, the constraint in Eq. (3c) must be satisfied. To address this issue, the cost function of the ECMS is modified as in Zhang \& Vahidi (2012), Serrao et al. (2011):

$$
\begin{aligned}
& \min _{u_{i}^{l}} \dot{m}_{e q v}(t) \Delta t=\left[\dot{m}_{f}\left(u_{i}^{l}(t)\right)+\right. \\
& \left.s^{e q v}(t)\left[\frac{\gamma}{\eta_{e l}\left(P_{b a t t}\right)}+(1-\gamma) \eta_{e l}\left(P_{b a t t}\right)\right] \frac{P_{b a t t}}{H_{L H V}}\right] \Delta t \\
& \gamma=\frac{1+\operatorname{sgn}\left(P_{b a t t}\right)}{2} \\
& \quad S O C\left(t_{f}\right)-\operatorname{SOC}(0) \leq \epsilon_{0}
\end{aligned}
$$

In the above equation, $\dot{m}_{\text {eqv }}(t)$ is the rate of equivalent fuel consumption, $s^{e q v}(t)$ is the equivalent factor, $\gamma$ is unit step function and $\eta_{e l}$ is the efficiency of the electrical path. The constraint in Eq. (3c) has been relaxed so that the difference between the final SOC and the initial SOC stays within a small bound $\epsilon_{0}$ as shown in Eq. (10b).

Since the equivalent factor $s^{e q v}(t)$ is a function of the co-states, it is also an optimization variable and the optimal solution is highly sensitive to the equivalent factor. In ECMS, the equivalent factor is either chosen as constant as in Zhang \& Vahidi (2012), Kim et al. (2011) or adaptive equivalent factor is used as in Onori et al. (2010), Musardo et al. (2005). The equivalent factor $s^{e q v}(t)$ can be considered to be constant only if the battery model parameters $V_{o c}$ and $R_{e q}$ do not change with time and the battery operates near a narrow range of SOC. Although with a good choice of equivalent factor have shown to provide acceptable solutions, adaptive ECMS has shown to provide better solutions as explained in Pisu \& Rizzoni (2007). In this paper, we use adaptive ECMS where the equivalent factor is chosen by the following way as mentioned in Onori et al. (2010):

$$
s^{e q v}(t+1)=0.5\left(s^{e q v}(t)+s^{e q v}(t-1)\right)+c_{p}(S O C(0)-S O C(t))
$$

In the above equation, $c_{p}$ is the step size, which acts as a proportional feedback gain as mentioned by Onori et al. (2010), and the equivalent factor $s^{e q v}(t)$ helps in the satisfaction of the constraint in Eq. (10b). It can be seen from Eq. (11) that the equivalent factor changes as the battery SOC deviates from its initial SOC $(S O C(0))$. The adaptive law is such that if the battery SOC at anytime $t$ is lower than its initial SOC, the equivalent factor $\left(s^{e q v}(t+1)\right)$ will increase, resulting in increase in the cost of battery power usage which will drive the solution to increase the battery SOC close to its initial SOC $(S O C(0))$. Similarly, if the battery SOC gets higher than its initial SOC, cost of battery power usage decreases, resulting in use of more battery power. The adaptive ECMS adds flexibility to the system as the solution does not completely depend on the initial equivalent factor and enables real-time operation.

For realistic simulation purposes, we utilize Autonomie software (Michaels et al. (2010)) which was developed as a collaborative effort between US Department of Energy, General Motors and Argonne National Laboratory. Autonomie is a powertrain and vehicle architecture development environment based on MATLAB/Simulink that supports plug-and-play and is widely used for the rapid evaluation of different technologies.

The overall integrated control strategy can be summarized as follows: 
- Each vehicle $i$ solves its higher level control problem using the recuperation efficiency obtained from the lower level controller. It transmits its state trajectory values over the horizon $T$ to the vehicle just behind it.

- The velocity profile for the horizon $T$ generated by the higher level controller is used as an input for its lower level controller.

- The input velocity from the higher level controller is mapped to power demand in the lower level and the problem in Eq. (10) is solved using adaptive ECMS, where the equivalent factor changes according to Eq. (11), to optimally split power between engine and the battery of the HEV.

- The Autonomie software provides the recuperation efficiency for a given horizon $T$ which is fed back to the higher level controller.

\section{SIMULATION RESULTS}

In this section, we present the simulation results of the methodology explained in Section 3. Our proposed method includes a hierarchical control strategy with higher level model predictive control and lower level adaptive ECMS strategy. Each vehicle implements the model predictive controller as defined by Eq. (8) so that: i) each vehicle minimizes its own average energy for traction, ii) avoids collision with other vehicles and iii) avoids stopping at red lights. To meet the power demand in an optimal manner, the power split between the engine and battery is performed by the adaptive ECMS strategy.

Detailed modeling of the HEVs are done using Autonomie software and the different parameters of the vehicles are described in the Tab. 1. For simulation purposes, we consider a scenarios with $n=10$ vehicles

Table 1: Components and Parameters of the Vehicle

\begin{tabular}{|c||c|}
\hline Component and Parameters & Description and Value \\
\hline Vehicle Mass $\left(M_{h}^{i}\right)$ & $990(\mathrm{~kg})$ \\
Vehicle Front Area $\left(A_{v}^{i}\right)$ & $2.25 \mathrm{~m}^{2}$ \\
Drag Co-efficient $\left(C_{D}\right)$ & 0.3 \\
\hline Engine & $110 \mathrm{~kW}$ and 2.2 L SI gasoline engine \\
\hline Motor & The permanent magnet electric motor of the \\
& MY04 with continuous power of 25kW and peak power of $50 \mathrm{~kW}$ \\
\hline Energy Storage & Li-ion Battery with capacity 6 Ah and 75 cells \\
\hline Transmission & 5 speed auto gearbox with final drive ratio 4.438 \\
\hline Power Converter & Output voltage 12V and efficiency 0.95 \\
\hline Wheel & P195/65 R15 \\
\hline
\end{tabular}

in a single lane road with traffic lights at every 500 meters. To emulate urban road conditions, the red $t_{r}$ and green light $t_{g}^{\text {original }}$ intervals are sampled from a uniform distribution with range 37 to 43 and 12 to 17 seconds respectively for each cycle of every traffic signal. As mentioned before, to address the noise associated with traffic light timings, the green light window $t_{g}$, used for evaluating the target velocity, is chosen less that the original window (as communicated though V2I) so that probability of passing through the traffic signal in that interval is 1 . Noise and delay associated with V2V communication is not considered in this paper. The maximum allowable velocity $v_{\max }$ is considered to be $20 \mathrm{~m} / \mathrm{s}$ while the minimum velocity is considered to be $v_{\text {min }}=0 \mathrm{~m} / \mathrm{s}$. The initial position of each vehicle is chosen randomly with initial relative distance between any two vehicles ranging from $15 \mathrm{~m}$ to $20 \mathrm{~m}$. The initial velocities of each vehicle are sampled from a uniform distribution ranging from $10 \mathrm{~m} / \mathrm{s}$ to $15 \mathrm{~m} / \mathrm{s}$.

We compare our approach with a baseline strategy where the vehicles do not use traffic light timing information and executes Connected Cruise Control (CCC) method (Jin \& Orosz (2014)) by sharing their instantaneous position, velocity and acceleration data. Unlike Jin \& Orosz (2014), we do not consider any delay in the system. For both our proposed method and the baseline method, the lower level control uses adaptive ECMS strategy. We run the simulation for 10 mins and the vehicle trajectories following our proposed method and the baseline method are shown in Fig. 6a and Fig. 6b respectively. A zoomed in version of the figures is shown in Fig. 7, where Fig. 7a and Fig. 7b shows the vehicle trajectories for 5 mins following the our proposed method and the baseline method respectively. In Fig 6 and 7, the horizontal red bars indicates the duration of red light windows while the blank spaces point to green light intervals through 
which vehicles can pass a given traffic signal. The velocity profile of each vehicle for both the proposed and the baseline method are shown in Fig. 8 and Fig. 9 respectively. It can be seen from the Fig 6a and Fig. 7a, that using our proposed method, none of the vehicles had to stop for red lights while the vehicles following the baseline method had to stop in all the traffic signals. This significantly improves the system mobility. The improvement of system mobility from our proposed method is also indicated by Fig. 8 where it can be seen that the vehicle velocities always stays positive (more that $5 \mathrm{~m} / \mathrm{s}$ ).

Table 2: Comparison between the Baseline Method and the Proposed Method

\begin{tabular}{|c|c|c|c|c|}
\hline Vehicle No. & \multicolumn{2}{|c|}{ Proposed Method } & \multicolumn{2}{c|}{ Baseline Method } \\
\hline & Fuel Efficiency (mpg) & $\mathrm{CO}_{2}$ Emission (gm/mile) & Fuel Efficiency $(\mathrm{mpg})$ & $\mathrm{CO}$ Emission $(\mathrm{gm} / \mathrm{mile})$ \\
\hline 1 & 49.89 & 181.00 & 31.80 & 282.71 \\
2 & 50.16 & 178.89 & 32.34 & 276.63 \\
3 & 50.27 & 177.56 & 33.54 & 267.01 \\
4 & 49.85 & 182.31 & 32.58 & 280.03 \\
5 & 51.44 & 172.79 & 31.90 & 280.67 \\
6 & 50.21 & 178.19 & 31.81 & 281.55 \\
7 & 51.06 & 175.29 & 32.12 & 27.02 \\
8 & 50.12 & 180.23 & 32.52 & 275.15 \\
9 & 50.85 & 176.13 & 33.78 & 269.35 \\
10 & 51.24 & 173.14 & 32.02 & 281.37 \\
\hline
\end{tabular}

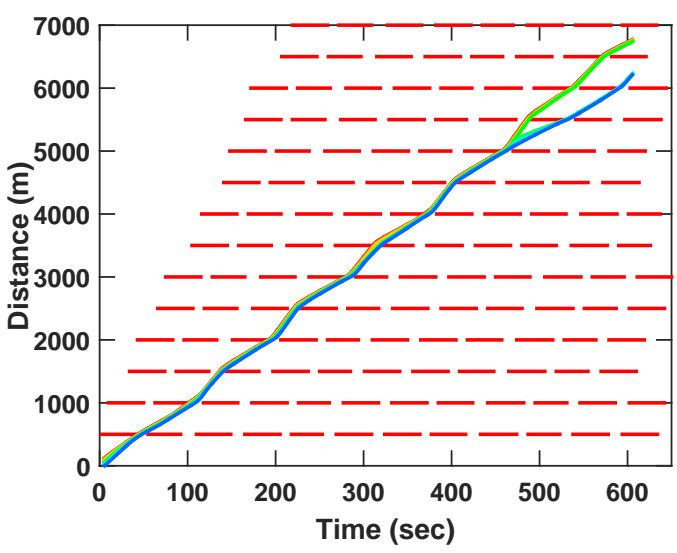

(a) Proposed Method

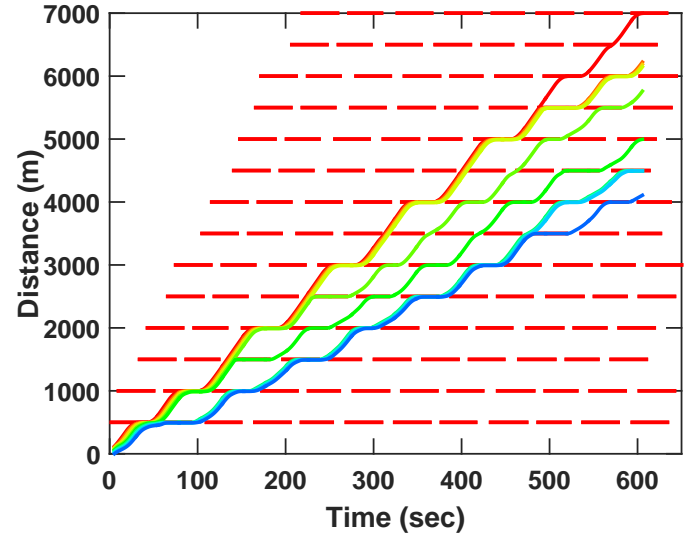

(b) Baseline Method

Figure 6: Comparison of Vehicle trajectories for 10 mins

For the lower level adaptive ECMS, the initial equivalent factor is chosen as $s^{e q v}(0)=2.5$ and it changes according to Eq. (11). The velocity tracking and the change of SOC with time when using adaptive ECMS in the lower level controller of all the 10 vehicles are shown in Fig. 11, Fig. 12, Fig. 13, Fig. 14, Fig. 15, Fig. 16, Fig. 17, Fig. 18, Fig. 19 and Fig. 20. It can be seen from the figures, that the lower level controller of all the vehicles is able to track the velocity profile supplied by the higher level controller almost perfectly and keeps the SOC of the battery within $\pm 1.5 \%$ of initial battery SOC. This is a very desirable performance of the lower level controller of all the vehicles.

The comparison between the baseline method and our proposed approach with respect to fuel efficiency and the $\mathrm{CO}_{2}$ emission for the 10 mins simulation is shown in Tab. 2. It can be seen from the results that our proposed method results in significant increase in fuel efficiency of individual vehicles as well as a significant reduction in $\mathrm{CO}_{2}$ emission. The fuel efficiency and the $\mathrm{CO}_{2}$ emission simulation data are generated by the Autonomie software for a given velocity profile and lower level control. The Tab. 2 along with Fig. $6 \mathrm{a}$ and Fig. 8 shows the improvement in system mobility, system fuel efficiency, and the improvement of environmental impact of the vehicles when using our proposed approach.

It can be seen from Fig. 6a that the group size and traffic light signal timings influences the behavior of the group as a number of vehicles (vehicles 7 to 10) chooses a different green light window to pass through a traffic signal after approximately 450 seconds resulting in bifurcation of the group. This can be seen in Fig. 8 


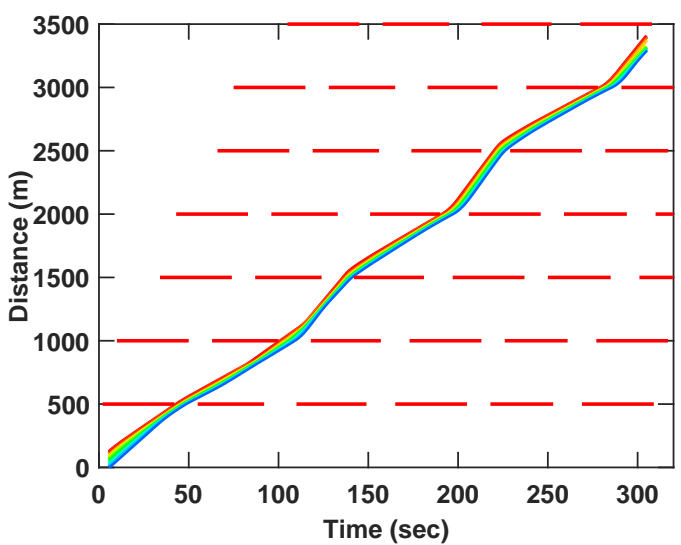

(a) Proposed Method

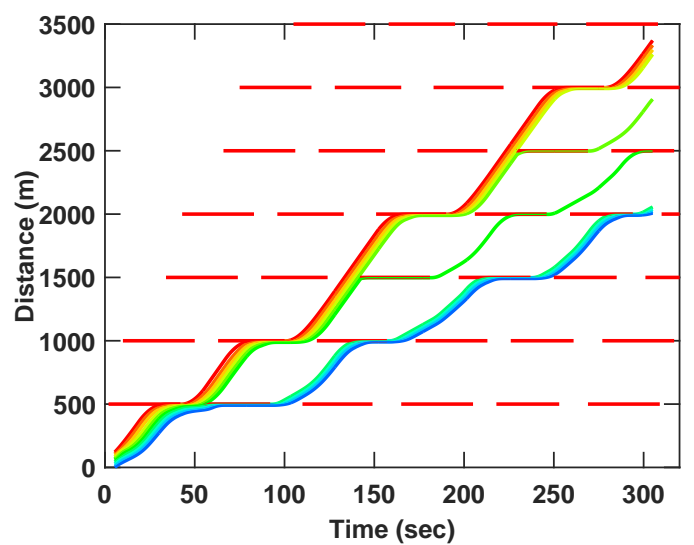

(b) Baseline Method

Figure 7: Comparison of Vehicle trajectories for 5 mins

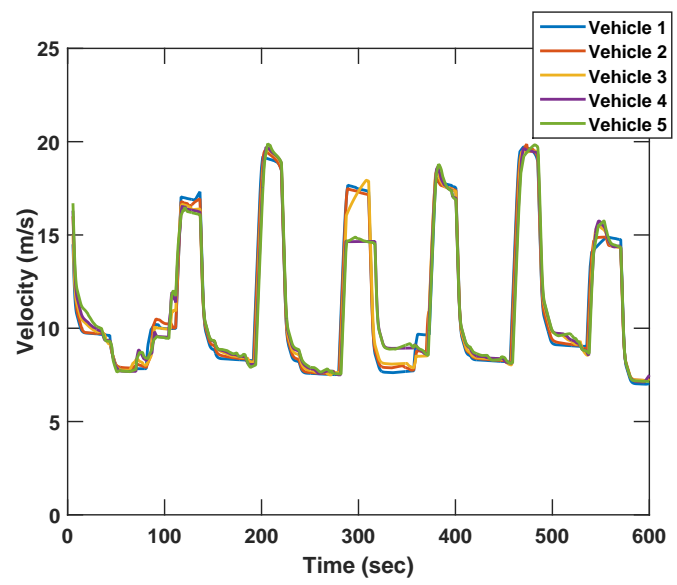

(a) Vehicle velocities for 1-5 Vehicles

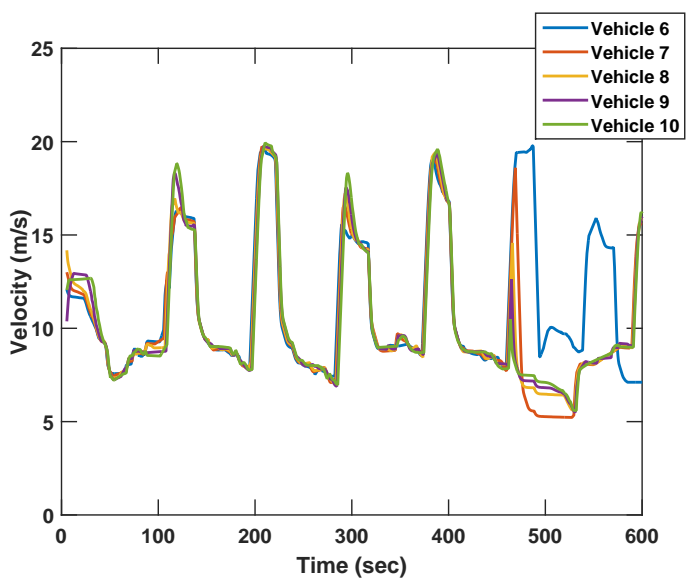

(b) Vehicle velocities for 6-10 Vehicles

Figure 8: Vehicle velocity profiles following the proposed method

where the vehicles 7 to 10 displays different velocity profile than the rest of the vehicles. It can me expected that as the number of vehicles increase, the whole group would be split into more number of smaller groups. In our simulation, the relative distance of the vehicles exhibiting similar vehicle profiles stays within $7 \mathrm{~m}$ to $10 \mathrm{~m}$.

Heterogeneous Scenario: We also present a scenario consisting of both regular and connected HEVs following our proposed strategy. Here, vehicles with index 5, 7 and 9 are considered not to be sharing V2V or V2I information and uses CCC methodology where they use instantaneous position, velocity and acceleration of the preceding connected vehicles. These position, velocity and acceleration data of the preceding vehicles can be considered to be measured by the regular HEVs (using radar sensors) rather than received through $\mathrm{V} 2 \mathrm{~V}$ communication. Now, the connected vehicles following our proposed methodology requires state trajectory information over the predicted horizon of the preceding vehicles. The vehicles just behind the regular HEVs (vehicle indexes 6, 8 and 10) do not have access to such state trajectory data of their preceding vehicle (because of no V2V communication) but can be considered to have access to their instantaneous position and velocity information using radar sensors. Thus a state trajectory of the preceding regular vehicle can be constructed assuming: (i) the preceding regular vehicle maintains the instantaneous velocity and (ii) the velocity of the preceding vehicle reduces at a constant rate, which is a very conservative 


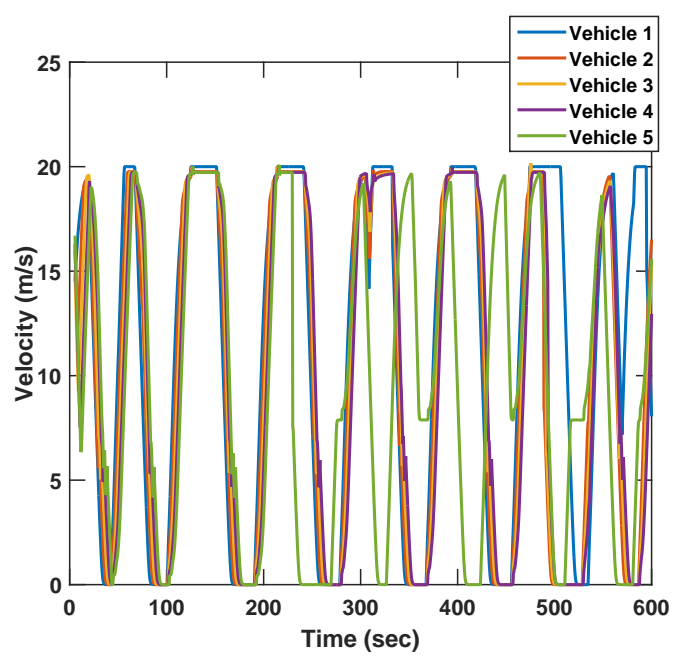

(a) Vehicle velocities for 1-5 Vehicles

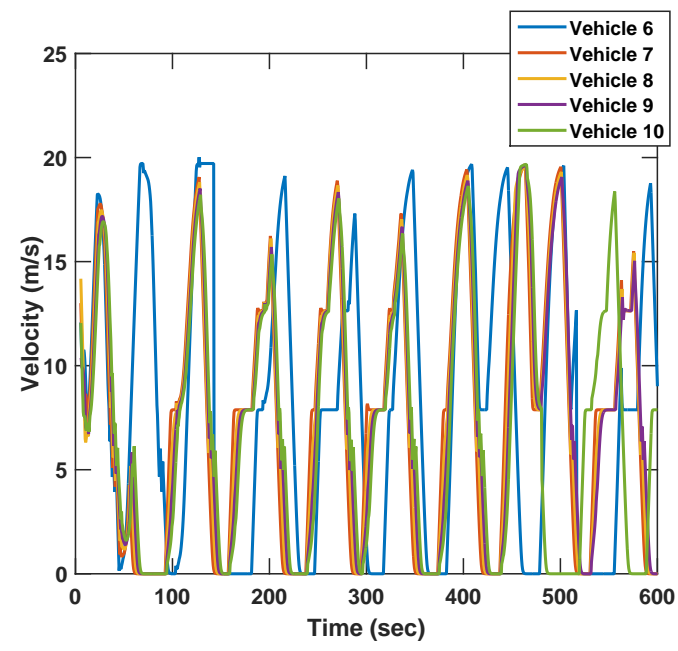

(b) Vehicle velocities for 6-10 Vehicles

Figure 9: Vehicle velocity profiles following the baseline method (CCC)

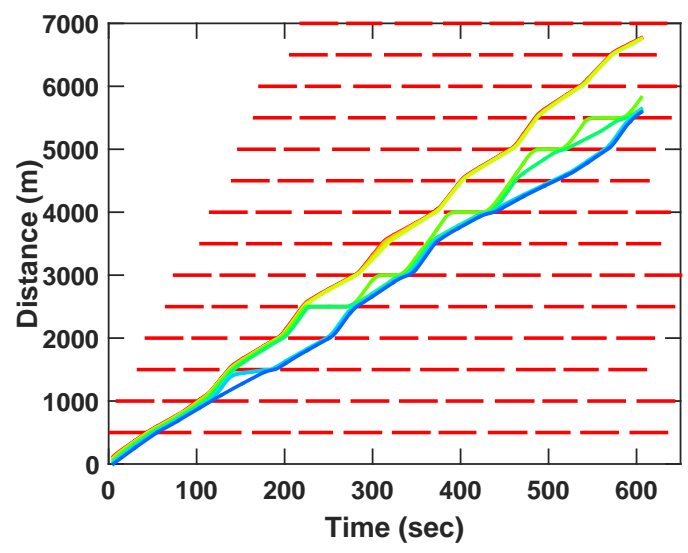

Figure 10: Vehicle trajectories in the heterogeneous scenario with vehicle index 5, 7 and 9 are regular HEVs

assumption. For the connected vehicles following another connected vehicle, the original state trajectory of the preceding vehicle is available and hence they can solve their problem without any issue.

In our simulation scenario, it has been seen that the vehicle velocities change a lot to address rear end collision and red light idling avoidance. Thus the state trajectory construction with the first assumption has been seen to result in vehicle collision. Using the second assumption of preceding vehicle state construction, Fig. 10 shows the vehicle trajectories for the heterogeneous scenario. Tab. 3 shows the simulation results where 'Regular' vehicle type refers to vehicles not using V2V or V2I information (uses instantaneous measurements of preceding vehicle) and follows CCC strategy while 'Connected' vehicle type indicates vehicles following our proposed method and uses information exchange. Connected vehicles with index 6, 7 and 10 performs the mentioned conservative state trajectory construction of the regular vehicles. It can be seen in Fig. 10 that vehicle index 5, since it follows a connected vehicle (which tries to avoid red light idling), is able to avoid first four traffic signals but has to stop at the fifth and the subsequent traffic signals (unable to follow vehicle 4). This is why vehicle 5 has the worst fuel efficiency and $\mathrm{CO}_{2}$ emission. Vehicle index 6 since it follows a regular vehicle has to stop at the fifth traffic signal (because of 'Regular' vehicle 5) but does not have to stop at the subsequent traffic signal as it chooses its target velocity in an intelligent manner. Now, we can see from the simulation results that the regular vehicles with index 7 and 9 does not stop at any traffic light, because they are able to follow connected vehicles that are avoiding stopping at red lights, and their 
fuel efficiency and $\mathrm{CO}_{2}$ emission are better than that of the ones in Tab. 2. This is due to the fact that they are avoiding red light idling and following optimal velocity profiles of the connected vehicles. Although their performance is better that the Baseline case, their fuel efficiency and $\mathrm{CO}_{2}$ emissions are inferior than that of the connected vehicles as they are not solving any optimization problem. It has been observed in a number of simulations using heterogeneous environment, that the relative distance between the connected and the regular vehicles is more than twice as much as between two connected vehicles. This is due to the fact that the connected vehicles has the information of the future state trajectory of their preceding vehicle and hence improved decision making is possible. The larger relative distance is one of the reason why vehicle index 5 was not able to cross the fifth traffic signal along with vehicle 4 . It may be noted that the performance of a heterogeneous group of vehicles would highly depend on the position and the number of regular vehicles in the group as well as on the traffic light signals. Overall, it has been observed that regular vehicles following connected vehicles has the potential of improving the performance of the regular vehicles.

Table 3: Heterogeneous Scenario with Both Regular and Connected Vehicles

\begin{tabular}{|c|c|c|c|}
\hline Vehicle No. & Vehicle Type & \multicolumn{2}{|c|}{ Vehicle Type } \\
\hline & & Fuel Efficiency (mpg) & $\mathrm{CO}_{2}$ Emission (gm/mile) \\
\hline 1 & Connected & 49.89 & 181.00 \\
2 & Connected & 50.16 & 178.89 \\
3 & Connected & 50.27 & 177.56 \\
4 & Connected & 49.85 & 182.31 \\
5 & Regular & 34.71 & 257.99 \\
6 & Connected & 45.65 & 209.14 \\
7 & Regular & 39.12 & 219.02 \\
8 & Connected & 47.78 & 187.41 \\
9 & Regular & 40.42 & 214.61 \\
10 & Connected & 48.17 & 185.89 \\
\hline
\end{tabular}


In this paper we have mainly focused on the development a hierarchical control strategy for a group of connected HEVs that aims at improving fuel efficiency and mobility of the group. The effectiveness of our proposed method is evident from the simulation results. To address more complex situations, such as noisy and delayed $\mathrm{V} 2 \mathrm{~V}$ and $\mathrm{V} 2 \mathrm{I}$ communication and presence of regular vehicles, stochastic and robust control strategies would be required along with learning and prediction algorithms.

\section{Acknowledgment}

Support for this research has been provided in part by the US Department of Energy GATE program. Any opinions, findings, and conclusions or recommendations expressed in this material are those of the author(s) and do not necessarily reflect the views of the US Department of Energy. 


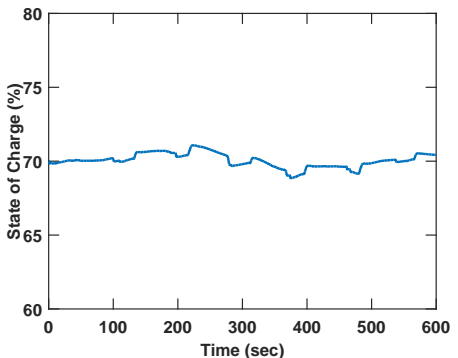

(a) Change of State of Charge

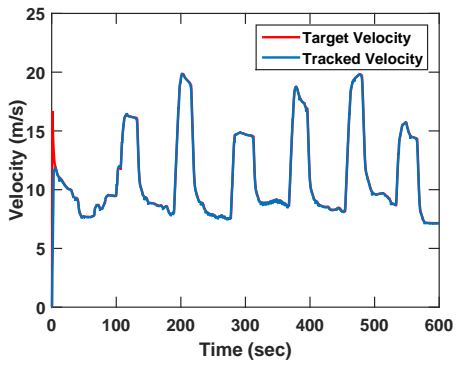

(b) Velocity Tracking by Lower Level Control

Figure 15: Lower Level Control: Vehicle 5

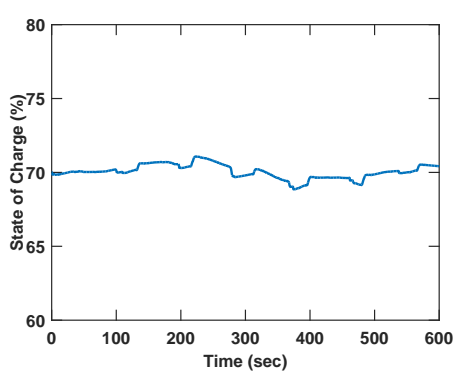

(a) Change of State of Charge

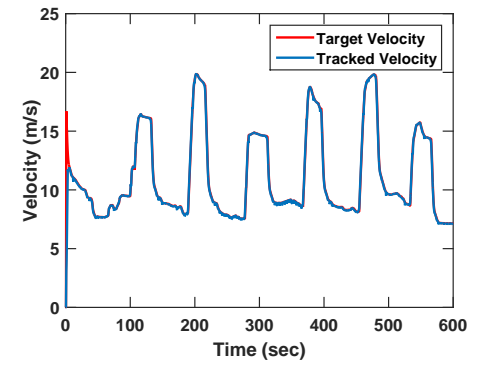

(b) Velocity Tracking by Lower Level Control

Figure 16: Lower Level Control: Vehicle 6

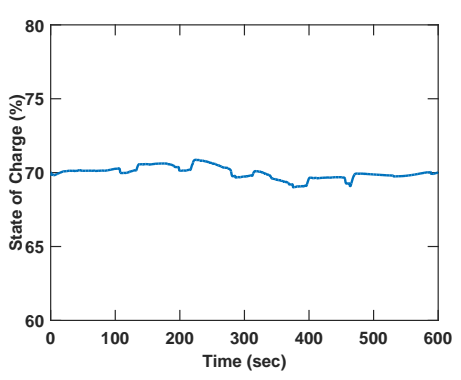

(a) Change of State of Charge

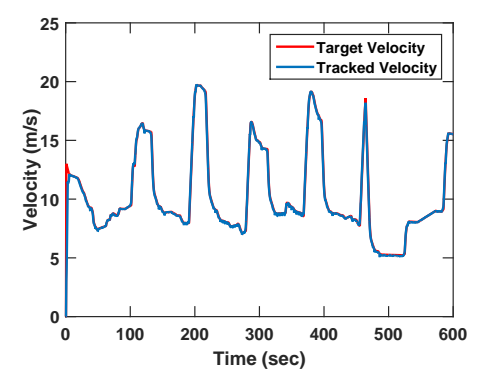

(b) Velocity Tracking by Lower Level Control

Figure 17: Lower Level Control: Vehicle 7 


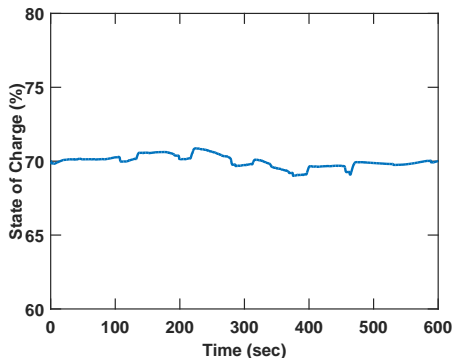

(a) Change of State of Charge

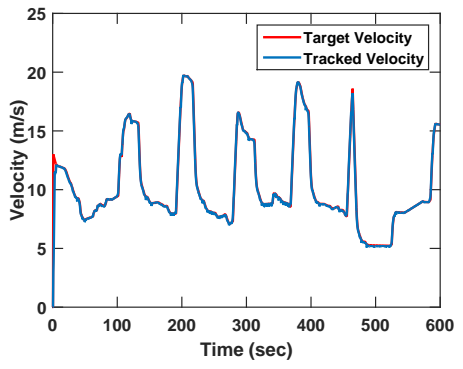

(b) Velocity Tracking by Lower Level Control

Figure 18: Lower Level Control: Vehicle 8

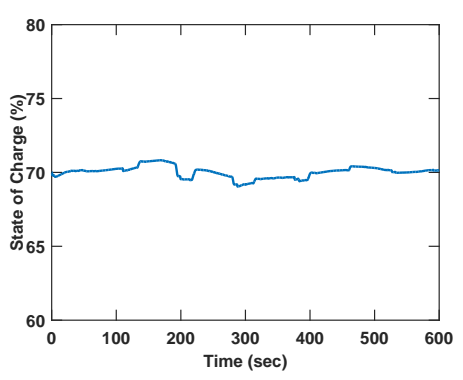

(a) Change of State of Charge

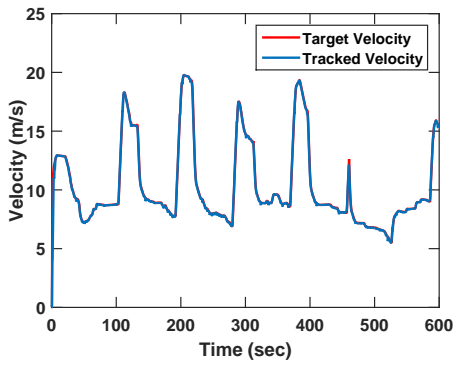

(b) Velocity Tracking by Lower Level Control

Figure 19: Lower Level Control: Vehicle 9

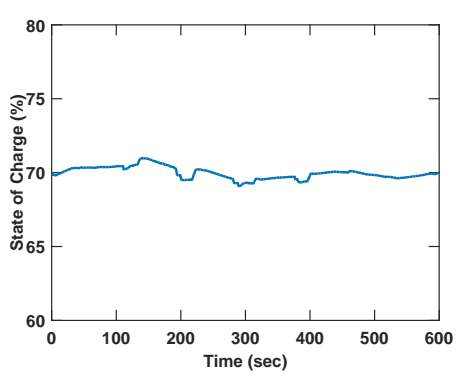

(a) Change of State of Charge

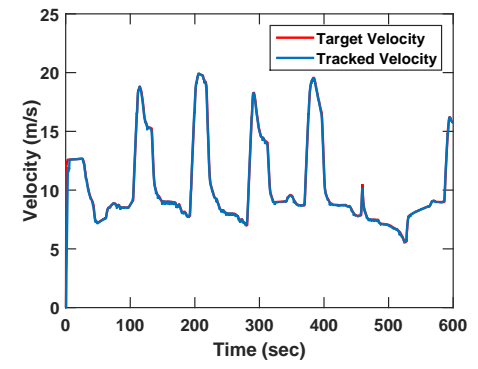

(b) Velocity Tracking by Lower Level Control

Figure 20: Lower Level Control: Vehicle 10 


\section{CONCLUSIONS}

In this paper, a decentralized fuel economic control strategy is presented for a group of HEVs that focuses on (i) improvement of fuel economy of the individual vehicles as well as for the group; (ii) reduction of red light idling; (iii) reduction of $\mathrm{CO}_{2}$ emission; and (iv) improvement of system mobility. The proposed method considers a connected vehicle scenario so that the Signal Time and Phase information of the traffic signals are considered to be available to the individual vehicles. Apart from that, the information of the states of the vehicles in the neighborhood of a vehicle is considered to be known knowledge. The paper proposes a novel hierarchical control architecture where both the higher and lower level control, although solves two different problems, aims at improving fuel efficiency of the vehicle and in the process improves some other aspects of the transportation system such as system mobility and $\mathrm{CO}_{2}$ emission. The simulation results presented in Section 4 shows the improvement in the performance of the individual vehicles when the proposed control method is utilized. This methodology can be used in full autonomous or semi-autonomous vehicles or as a driver assistance framework where the driver is considered to follow all the control instructions from the higher level controller. Some of the future research direction involves developing the higher level controller strategies in a driver-in-the-loop framework where the driver ability would be considered in the development of control strategies. This would be followed by the development of control strategies using prediction of future driver behavior. 


\section{References}

Asadi, B. \& Vahidi, A. (2011), 'Predictive cruise control: Utilizing upcoming traffic signal information for improving fuel economy and reducing trip time', Control Systems Technology, IEEE Transactions on 19(3), 707-714.

Boggs, P. T. \& Tolle, J. W. (1995), 'Sequential quadratic programming', Acta numerica 4, 1-51.

Brahma, A., Guezennec, Y. \& Rizzoni, G. (2000), Optimal energy management in series hybrid electric vehicles, in 'American Control Conference, 2000. Proceedings of the 2000', Vol. 1, IEEE, pp. 60-64.

Camacho, E. F. \& Alba, C. B. (2013), Model predictive control, Springer Science \& Business Media.

Chang, D. J. \& Morlok, E. K. (2005), 'Vehicle speed profiles to minimize work and fuel consumption', Journal of transportation engineering 131(3), 173-182.

De Nunzio, G., Canudas de Wit, C., Moulin, P. \& Di Domenico, D. (2013), Eco-driving in urban traffic networks using traffic signal information, in 'Decision and Control (CDC), 2013 IEEE 52nd Annual Conference on', IEEE, pp. 892-898.

Geering, H. P. (2007), Optimal control with engineering applications, Springer.

Gong, Q., Li, Y. \& Peng, Z.-R. (2008), 'Trip-based optimal power management of plug-in hybrid electric vehicles', Vehicular Technology, IEEE Transactions on 57(6), 3393-3401.

Grüne, L. \& Pannek, J. (2011), Nonlinear model predictive control, Springer.

Guzzella, L. \& Sciarretta, A. (2007), Vehicle propulsion systems, Vol. 1, Springer.

He, X., Liu, H. X. \& Liu, X. (2015), 'Optimal vehicle speed trajectory on a signalized arterial with consideration of queue', Transportation Research Part C: Emerging Technologies 61, 106-120.

HomChaudhuri, B., Vahidi, A. \& Pisu, P. (2015), A fuel economic model predictive control strategy for a group of connected vehicles in urban roads, in 'accepted in American Control Conference'.

Jin, I. G. \& Orosz, G. (2014), 'Dynamics of connected vehicle systems with delayed acceleration feedback', Transportation Research Part C: Emerging Technologies 46, 46-64.

Kamal, M., Mukai, M., Murata, J. \& Kawabe, T. (2010), 'Ecological driver assistance system using modelbased anticipation of vehicle-road-traffic information', IET intelligent transport systems 4(4), 244-251.

Kamal, M. S., Mukai, M., Murata, J. \& Kawabe, T. (2013), 'Model predictive control of vehicles on urban roads for improved fuel economy', Control Systems Technology, IEEE Transactions on 21(3), 831-841.

Kim, N., Cha, S. \& Peng, H. (2011), 'Optimal control of hybrid electric vehicles based on pontryagin's minimum principle', Control Systems Technology, IEEE Transactions on 19(5), 1279-1287.

Lin, C.-C., Kang, J.-M., Grizzle, J. \& Peng, H. (2001), Energy management strategy for a parallel hybrid electric truck, in 'American Control Conference, 2001. Proceedings of the 2001', Vol. 4, IEEE, pp. 28782883.

Mahler, G. \& Vahidi, A. (2014), 'Red light avoidance through probabilistic traffic signal timing prediction', IEEE Transactions on Intelligent Transportation Systems 15, 2516- 2523.

Mandava, S., Boriboonsomsin, K. \& Barth, M. (2009), Arterial velocity planning based on traffic signal information under light traffic conditions, in 'Intelligent Transportation Systems, 2009. ITSC'09. 12th International IEEE Conference on', IEEE, pp. 1-6.

Mensing, F., Bideaux, E., Trigui, R., Ribet, J. \& Jeanneret, B. (2014), 'Eco-driving: An economic or ecologic driving style?', Transportation Research Part C: Emerging Technologies 38, 110-121. 
Michaels, L., Pagerit, S., Rousseau, A., Sharer, P., Halbach, S., Vijayagopal, R., Kropinski, M., Matthews, G., Kao, M., Matthews, O. et al. (2010), Model-based systems engineering and control system development via virtual hardware-in-the-loop simulation, Technical report, SAE Technical Paper.

Musardo, C., Rizzoni, G., Guezennec, Y. \& Staccia, B. (2005), 'A-ecms: An adaptive algorithm for hybrid electric vehicle energy management', European Journal of Control 11(4), 509-524.

Najm, W. G., Koopmann, J., Smith, J. D. \& Brewer, J. (2010), Frequency of target crashes for intellidrive safety systems, Technical report, U.S. Department of Transportation National Highway Traffic Safety Administration.

Onori, S., Serrao, L. \& Rizzoni, G. (2010), Adaptive equivalent consumption minimization strategy for hybrid electric vehicles, in 'ASME 2010 Dynamic Systems and Control Conference', American Society of Mechanical Engineers, pp. 499-505.

Paganelli, G., Ercole, G., Brahma, A., Guezennec, Y. \& Rizzoni, G. (2001), 'General supervisory control policy for the energy optimization of charge-sustaining hybrid electric vehicles', JSAE review 22(4), 511518.

Paganelli, G., Guerra, T., Delprat, S., Santin, J., Delhom, M. \& Combes, E. (2000), 'Simulation and assessment of power control strategies for a parallel hybrid car', Proceedings of the Institution of Mechanical Engineers, Part D: Journal of Automobile Engineering 214(7), 705-717.

Pisu, P. \& Rizzoni, G. (2007), 'A comparative study of supervisory control strategies for hybrid electric vehicles', Control Systems Technology, IEEE Transactions on 15(3), 506-518.

Qin, S. J. \& Badgwell, T. A. (2003), 'A survey of industrial model predictive control technology', Control engineering practice 11(7), 733-764.

Salman, M., Schouten, N. J. \& Kheir, N. A. (2000), Control strategies for parallel hybrid vehicles, in 'American Control Conference, 2000. Proceedings of the 2000', Vol. 1, IEEE, pp. 524-528.

Sciarretta, A. \& Guzzella, L. (2007), 'Control of hybrid electric vehicles', Control systems, IEEE 27(2), 6070.

Serrao, L., Onori, S. \& Rizzoni, G. (2009), Ecms as a realization of pontryagin's minimum principle for hev control, in 'Proceedings of the American control conference', pp. 3964-3969.

Serrao, L., Onori, S. \& Rizzoni, G. (2011), 'A comparative analysis of energy management strategies for hybrid electric vehicles', Journal of Dynamic Systems, Measurement, and Control 133(3), 031012.

Sun, C., Moura, S. J., Hu, X., Hedrick, J. K. \& Sun, F. (2015), 'Dynamic traffic feedback data enabled energy management in plug-in hybrid electric vehicles', Control Systems Technology, IEEE Transactions on 23(3), 1075-1086.

Treiber, M., Hennecke, A. \& Helbing, D. (2000), 'Congested traffic states in empirical observations and microscopic simulations', Physical Review E 62(2), 1805.

Van Mierlo, J., Maggetto, G., Van de Burgwal, E. \& Gense, R. (2004), 'Driving style and traffic measuresinfluence on vehicle emissions and fuel consumption', Proceedings of the Institution of Mechanical Engineers, Part D: Journal of Automobile Engineering 218(1), 43-50.

Vogel, A., Ramachandran, D., Gupta, R. \& Raux, A. (2012), Improving hybrid vehicle fuel efficiency using inverse reinforcement learning., in 'AAAI'.

Wang, Y. \& Boyd, S. (2010), 'Fast model predictive control using online optimization', Control Systems Technology, IEEE Transactions on 18(2), 267-278.

Yang, H. \& Jin, W.-L. (2014), 'A control theoretic formulation ofgreen driving strategies based on intervehicle communications', Transportation Research Part C: Emerging Technologies 41, 48-60. 
Zhang, C. \& Vahidi, A. (2011), Predictive cruise control with probabilistic constraints for eco driving, in 'ASME 2011 Dynamic Systems and Control Conference and Bath/ASME Symposium on Fluid Power and Motion Control', American Society of Mechanical Engineers, pp. 233-238.

Zhang, C. \& Vahidi, A. (2012), 'Route preview in energy management of plug-in hybrid vehicles', Control Systems Technology, IEEE Transactions on 20(2), 546-553.

Zhang, C., Vahidi, A., Li, X. \& Essenmacher, D. (2009), Role of trip information preview in fuel economy of plug-in hybrid vehicles, in 'ASME 2009 Dynamic Systems and Control Conference', American Society of Mechanical Engineers, pp. 253-258.

Zhang, C., Vahidi, A., Pisu, P., Li, X. \& Tennant, K. (2010), 'Role of terrain preview in energy management of hybrid electric vehicles', Vehicular Technology, IEEE Transactions on 59(3), 1139-1147.

Zhang, X. \& Mi, C. (2011), Vehicle power management: modeling, control and optimization, Springer Science \& Business Media. 